A N N A L E S Annales de Bretagne et des Pays de l'Ouest

\title{
Les prises d'armes de 1814-1815, signe et facteur de la politisation des ruraux
}

The uprising of 1814-1815, a sign and factor of the politicization of the rural population

\section{Jacques Hantraye}

\section{(2) OpenEdition}

\section{Journals}

Édition électronique

URL : http://journals.openedition.org/abpo/2135

DOI : 10.4000/abpo.2135

ISBN : 978-2-7535-1841-4

ISSN : 2108-6443

\section{Éditeur}

Presses universitaires de Rennes

\section{Édition imprimée}

Date de publication : 30 décembre 2011

Pagination : 47-61

ISBN : 978-2-7535-1839-1

ISSN : 0399-0826

\section{Référence électronique}

Jacques Hantraye, "Les prises d'armes de 1814-1815, signe et facteur de la politisation des ruraux », Annales de Bretagne et des Pays de l'Ouest [En ligne], 118-4 | 2011, mis en ligne le 30 décembre 2013, consulté le 30 avril 2019. URL : http://journals.openedition.org/abpo/2135; DOI : 10.4000/abpo.2135 


\title{
Les prises d'armes de 1814-1815, signe et facteur de la politisation des ruraux
}

\author{
Jacques HANTRAYE \\ Professeur agrégé d'histoire au lycée Guillaume-Budé de Limeil-Brévannes \\ (Val-de-Marne) \\ docteur en histoire, chercheur associé au Centre d'histoire du XIX ${ }^{\mathrm{e}}$ siècle \\ (EA 3550/Paris-Sorbonne)
}

Les invasions et les occupations de la fin du Premier Empire ont longtemps constitué des moments méconnus dans l'histoire des prises d'armes de la période contemporaine. L'étude des guérillas du début du XIXe siècle, menée par les historiens français et étrangers dans un cadre européen, apporte un éclairage nouveau sur ces pratiques de guerre. On pense en particulier aux travaux récents des Anglo-saxons et des Français sur l'Espagne ou la Calabre, sans oublier que cette forme d'action a été également conduite dans le Tyrol, mais aussi par des combattants serbes contre les Turcs en 1804 et en Finlande contre les Russes en 1808-1809¹.

Afin de comprendre les principaux enjeux des prises d'armes en France, il convient de rappeler brièvement dans quel cadre elles se déroulent. La France est envahie à deux reprises. Des unités constituées de Britanniques, d'Espagnols et de Portugais combattent dans le Sud-Ouest entre octobre 1813 et avril 1814. En parallèle, à la fin de décembre 1813, les armées des États allemands et des empires d'Autriche et de Russie traversent le Rhin, tandis que des opérations sont menées de la FrancheComté aux Alpes. L'abdication de Napoléon et l'avènement de Louis XVIII ont lieu peu après la chute de Paris le 31 mars 1814. Les armées étrangères se retirent après la signature du traité de Paris le 30 mai 1814. L'année

1. EsDallE, Charles, "Popular Resistance in Napoleonic Europe : Issues and Perspectives ", dans EsDalle, Charles (dir.), Popular Resistance in the French Wars, Houndsmill, Palgrave MacMillan, 2005, p. 204; CADET, Nicolas, "Anatomie d'une "petite guerre", la campagne de Calabre de 1806-1807 ", Revue d'histoire du XIXe siècle, n 30, 2005, p. 65-84; Lafon, Jean-Marc, L'Espagne aux XIX et XXe siècles, Paris, Ellipses, 2007 et HoCQUELLET, R., Résistance et Révolution durant l'occupation napoléonienne en Espagne 1808-1812, Paris, La Boutique de l'Histoire, 2001. 
suivante, la fuite de Napoléon de l'île d'Elbe soude à nouveau les puissances européennes. Après la victoire des Alliés à Waterloo le 18 juin 1815, Louis XVIII rentre dans la capitale le 8 juillet suivant. Les Alliés pénètrent un peu dans le secteur pyrénéen, et plus largement à travers les frontières de l'Est, du Nord aux Alpes. Le traité de paix est signé le 20 novembre 1815. Dans ce contexte, quelle est la dimension politique des prises d'armes? Dans quelle mesure sont-elles spontanées? Enfin, ce mouvement s'inscrit-il dans la durée ou s'interrompt-il avec le changement de régime? Faute de pouvoir répondre complètement à ces questions, on se contentera de faire état de quelques pistes qui restent à approfondir, en s'intéressant plus particulièrement aux ruraux. On abordera en premier lieu les obstacles méthodologiques, avant de s'intéresser à la compréhension des levées. Enfin, on étudiera leur postérité sur le plan politique.

\section{Des sources rares}

Tout d'abord, signalons que les sources sont peu nombreuses et peu disertes, ce qui est un problème signalé par d'autres chercheurs européens $^{2}$. En effet, les prises d'armes ont lieu au cours de périodes de crise, moments pendant lesquels les administrateurs ont d'autres préoccupations, y compris leur propre sécurité et leur maintien en fonction. De plus, ils n'ont guère les moyens de s'informer au sujet d'organisations armées souvent marginales. Le texte d'un nommé Patel, employé de l'enregistrement à Pontarlier, est l'un des rares écrits à la première personne ${ }^{3}$. L'origine souvent modeste des combattants explique qu'ils n'aient guère pris la plume. Enfin, le contexte politique de la Restauration a conduit les acteurs à occulter la part prise à ces actions. D'ailleurs, certains militaires manifestent du mépris à l'égard de cette forme de combat, comme l'ancien commissaire des guerres Joseph Pellot qui qualifie en 1818 les combattants irréguliers des Pyrénées en 1814 et 1815 de " dogues " vivant sur l'habitant ${ }^{4}$. Le plus souvent, les militaires étrangers les dédaignent. En Lorraine, le feld-maréchal prince de Wrède les qualifie de "canaille armée " et de brigands ${ }^{5}$. Dans le même temps, ils les craignent. Dans l'Aisne, en février 1814, un officier prussien redoute ainsi que la situation n'évolue comme en Espagne ${ }^{6}$.

2. EsDAlle, Charles, Fighting Napoleon, New Haven, Yale University Press, 2004, p. 195196.

3. Patel, Souvenirs des deux invasions de 1814 et 1815 dans l'arrondissement de Pontarlier, Pontarlier, Simon, 1865.

4. Pellot, Joseph, Mémoire sur la campagne de l'armée française dite des Pyrénées, Bayonne, Gosse, 1818, p. 122-123.

5. Les corps francs du commandant Brice en Lorraine, Vitry-le-François, Bitsch, 1868, p. 7 et 12 .

6. FLEURY, Édouard, Le Département de l’Aisne en 1814, Laon, Fleury, 1858, p. 169-170. 


\section{Les prises d'armes et leurs auteurs}

Ces levées interviennent lors de l'invasion. L'initiative en revient en partie au pouvoir impérial. À la fin de 1813, les troupes régulières ayant fondu, on décide de faire appel à la garde nationale, en sommeil depuis la fin de la Révolution. Ce corps comprend deux catégories : la garde nationale sédentaire, chargée de la police, voire en dernier recours de la défense des communes, et la garde nationale en activité, qui peut être appelée à se battre aux côtés de l'armée, y compris hors de son département d'origine. La seconde est convoquée plus tôt que la première, par un décret du 17 décembre $1813^{7}$. Elle est recrutée dans le cadre communal, puis regroupée à l'échelon départemental dans 17 départements suivant les décrets du 30 décembre 1813 et du 6 janvier 1814. Il s'agit de former deux armées de réserve couvrant Paris et Lyon ${ }^{8}$. Ces unités rassemblent environ 40000 hommes au début de $1814^{9}$. Elles combattent avec un succès inégal. À Fère-Champenoise, le 25 mars 1814, on loue l'action des gardes nationaux de la division Pacthod. Mais dans le même temps sont rassemblés à Meaux des conscrits et des gardes nationaux non formés et mal commandés ${ }^{10}$. En 1815, ces troupes sont affectées à la défense des places fortes proches des frontières.

Ces effectifs sont certes insuffisants pour renforcer l'armée, mais ils ne sont pas insignifiants si l'on envisage la mobilisation sous l'angle de la politisation des civils. Toutefois, notons que la garde nationale est très encadrée par le pouvoir. Aussi ne peut-on prendre en compte d'un point de vue politique que le rejet éventuel, qui se manifeste par l'insoumission ou la désertion. Encore faut-il interpréter cette attitude avec précaution car la garde nationale comprend une proportion importante de remplaçants.

Quelques semaines après la mise en activité de la garde nationale mobilisée en décembre 1813, on organise la garde nationale sédentaire entre janvier et mars 1814. À cette date, la situation militaire devient préoccupante et le pouvoir doit se résoudre à lever également des corps francs par le décret du 4 janvier, puis à ordonner la levée en masse par les décrets de Fismes, dont celui du 5 mars $1814^{11}$. Il s'agit d'un moment où l'adhésion et la spontanéité devraient être plus évidentes. Les troupes qui s'organisent alors se trouvent à la charnière de l'armée régulière, des unités auxiliaires comme la garde nationale, et des soulèvements plus ou moins spontanés. On peut citer pour mémoire les unités de gardes forestiers, de douaniers ou encore de gardes champêtres dont le rôle est de surveiller les forêts et

7. GIRARD, Louis, La Garde nationale 1814-1871, Paris, Plon, 1964, p. 39.

8. CARROT, Georges, La Garde nationale 1789-1871, thèse de $3^{\mathrm{e}}$ cycle, université de Nice, 1979, p. 132.

9. Houssaye Henry, 1814, Paris, Perrin, 1894, p. 11.

10. Ibidem, p. $380-381$ et 386 .

11. Bulletin des Lois, ${ }^{\circ} 12,4$ janvier 1814 et $n^{\circ} 562,5$ mars 1814.

Dans les départements frontaliers du Nord-Est en 1814, par exemple dans l'Aisne, on trouve deux levées en masse, par une proclamation du préfet du 23 janvier 1814, suivie d'un autre appel au mois de mars (FLEURY Édouard, Le Département de l'Aisne..., op. cit., p. 31-33). 
de capturer les ennemis égarés près des zones de combat. On les retrouve dans cette même fonction en 1815. Il leur arrive d'être convoqués individuellement, ce qui ôte beaucoup de spontanéité à leur engagement ${ }^{12}$. Ces hommes sont placés sous l'autorité de leurs supérieurs hiérarchiques ou bien de notables locaux, comme Jean-Baptiste Drouet, sous-préfet de Sainte-Menehould - dans la Marne -, au début de $1814^{13}$.

Les hommes des corps francs, recrutés dans les secteurs directement menacés par l'ennemi, sont souvent d'anciens militaires rassemblés par des officiers qui en ont reçu l'autorisation de la part de l'empereur, d'un préfet ou d'un général. Dans les Vosges, Nicolas Wolff obtient l'accord du préfet de la Meurthe, le baron de Flégny ${ }^{14}$. Dans le Morvan, le maire d'Alligny consulte le sous-préfet de Château-Chinon en février $1814^{15}$. Ces troupes, appelées parfois " compagnie franche " ou " compagnie de partisans ", sont équipées et armées aux frais de leur commandant ou des combattants eux-mêmes ${ }^{16}$.

On compte beaucoup sur la personnalité du chef, comme l'écrit le duc de Feltre le 20 février $1814^{17}$. Ainsi, le chef d'escadron Joseph Nicolas Brice, dans la Meurthe, met en avant son prestige d'officier et se fait accompagner dans sa tournée de recrutement par un cavalier vêtu en mamelouk ${ }^{18}$. Les corps francs révèlent l'existence de réseaux : dans les Vosges, le maître de forges Wolff, par exemple, réunit une partie de ses ouvriers, tandis qu'un maître d'école qui lutte sous ses ordres commande lui-même ses élèves au combat ${ }^{19}$. En 1814, Nicolas Jacques Frantz, de Metz, assemble une « compagnie de partisans " de 44 cavaliers. L'année suivante, il forme le $2^{\mathrm{e}}$ corps franc de la Moselle, qui comprend plus de 600 hommes. Il y a beaucoup d'anciens militaires parmi eux, dont 48 officiers décorés de la Légion d'honneur ${ }^{20}$.

L'origine sociale des chefs est variée, mais à côté d'un Joseph Brice, fils d'un instituteur lorrain ${ }^{21}$, ils appartiennent en majorité à des familles de notables. Beaucoup sont des militaires appartenant aux promotions de la fin de l'Empire. Présentons brièvement quelques parcours. Charles-Nicolas

12. Arch. dép. de l'Aisne, A C Crépy, 4 H 2, sous-préfet de Laon au maire de Crépy, 3.6.1815.

13. ClaUSE, Georges, " 1814 : la Champagne entre les armées et les pouvoirs ", dans : BERCÉ Yves-Marie (dir.), La fin de l'Europe napoléonienne. La vacance du pouvoir, Paris, Veyrier, 1990, p. 264.

14. NERLINGER, Charles, Nicolas Wolff et la défense des Vosges 1814-1815, Strasbourg, StaatNoiriel, 1897, p. 14.

15. Brosselin-Thomas, Arlette, "Les "maquis" de Bourgogne lors des invasions de 18141815 ", dans : CORVOL Andrée et AMAT, Jean-Pierre (dir.), Forêt et guerre, Paris, L'Harmattan, 1994, p. 97.

16. PATEL, Souvenirs des deux invasions..., op. cit., p. 36 et 38.

17. Chambon, Pascal, La Loire et l'aigle, Saint-Etienne, Presses universitaires de SaintEtienne, 2005, p. 386.

18. Les corps francs..., op. cit., p. 6.

19. NERLINGER, Charles, Nicolas Wolff..., op. cit., p. 7 et 18.

20. Pétition du capitaine Frantz au prince Louis-Napoléon Bonaparte président de la République, Paris, Pommeret, 1851, p. 3.

21. Les corps francs..., op. cit., p. 24. 
Fabvier (1782-1855), originaire de Pont-à-Mousson, est le fils d'un avocat. Elève à Polytechnique, il devient colonel et baron de l'Empire à 31 ans $^{22}$. Frantz, capitaine de corps franc en 1815, est né à Sarrelouis, alors française, dans une famille riche. Il est avocat à la fin de l'Empire ${ }^{23}$. Nicolas Wolff (1761-1846), riche maître de forges et marchand de bois de Rothau, dans les Vosges, est un ancien militaire devenu maire de sa commune ${ }^{24}$. Ailleurs, c'est un officier supérieur originaire du pays qui entraîne l'adhésion. Dans les Pyrénées, on soutient le général Jean-Isidore Harispe, originaire de Saint-Etienne de Baïgorry. Cet ancien des corps francs de 1793, puis de la guerre d'Espagne à partir de 1808, commande les corps francs du Sud-Ouest en $1814^{25}$. Ces hommes appartiennent à toutes les générations de la seconde moitié du XVIII siècle, même si la majorité est née dans les années 1780. Ce sont donc principalement des enfants de la Révolution et de l'Empire. À l'inverse, le parcours de Florian dans le Sud Ouest en 1814 est exceptionnel. Il rassemble une vingtaine de cavaliers, dont un grand nombre d'étrangers, afin de harceler les Britanniques ${ }^{26}$. Curieux personnage, qui charge avec ses cavaliers au cri de "Vive l'empereur ", avant de se livrer au brigandage ${ }^{27}$. Lui et ses hommes apparaissent comme des militaires incapables de se réinsérer dans un contexte de sortie de guerre.

Le parcours des corps francs et les formes de combat qu'ils adoptent ne sont pas sans évoquer ce que l'on observe en Italie, en Espagne, en Allemagne, ou encore dans le Tyrol au cours des années précédentes, mais dans une certaine mesure seulement. En effet, ces troupes ne s'ancrent pas dans une tradition de brigandage comme en Calabre, ou n'incluent pas des étudiants dans leurs rangs comme les corps francs allemands ${ }^{28}$. Le rôle du clergé est très secondaire, à la différence de ce que l'on observe en Espagne $^{29}$. Enfin, ces prises d'armes ne s'inscrivent pas dans la durée, ne serait-ce que parce qu'elles interviennent à la fin des campagnes de 1814 et 1815. Ainsi, la Légion lyonnaise de chasseurs à pied et à cheval de Gustave de Damas, dans la Loire et les environs, ne se met en route que

22. DeBIDOUR, Antonin, Étude sur le général Fabvier, 1782-1855, Nancy, Berger-Levrault, 1885 , p. 6.

23. Pétition..., op. cit., p. 2.

24. NERLINGER, Charles, Nicolas Wolff..., op. cit., p. 7-9. Dans le centre-Est, Gustave de Damas (1788-1842), qui forma un corps franc en 1814, appartient à une famille de la vieille noblesse forézienne. Après avoir suivi ses parents en émigration, il revient en France en 1802 et sert dans l'armée impériale (ChAmBon, Pascal, La Loire ..., op. cit., p. 386 et Roman D’Amat (dir.), Dictionnaire de biographie française, Paris, Letouzey, 1965, p. 30-31).

25. LaUzun, Philippe, Florian et ses bandes de partisans en 1814 et 1815, Agen, imprimerie moderne, 1907, p. 4; PelLot, Joseph, Mémoire sur la campagne..., op. cit., p. 91.

26. Ibidem, p. 10-11.

27. Ibid., p. 14 et 30.

28. BOURGUINAT, Nicolas, "Entre brigandage et guérilla : maintien de l'ordre et répression dans l'Italie napoléonienne centrale et méridionale ", dans : PETITEAU, Nathalie (dir.), Conflits d'Empire, Les Cahiers du GERHICO, n 9, 2006, p. 15-16; Roux, David, « La résistance prussienne face à la présence française pendant les années 1807-1813 : le cas du corps de Lützow ", ibidem, p. 70.

29. EsDAlle, Charles, «Popular Resistance in Napoleonic Europe... », art. cit., p. 208. 
le 20 février $1814^{30}$. En Lorraine, le chef d'escadron Brice agit seulement entre le 24 juin et le 19 juillet $1815^{31}$. Cependant, il existe des points communs, comme les formes de combat, mais aussi les lieux concernés, ainsi les forêts ou les zones montagneuses ${ }^{32}$.

On a découvert récemment le caractère très " régulier " de certaines de ces formations, notamment en Espagne et en Calabre. Mais cet aspect est sans doute encore plus marqué en France. Dans leur façon de combattre et leur organisation, les corps francs s'inspirent de l'armée. Ainsi, dans la région de Pontarlier en 1815, gardes nationales et corps francs obéissent en dernier ressort au général Lecourbe ${ }^{33}$. Même la levée en masse est conçue de cette façon : lors de la défense de Laon en juin 1815, il est prévu de rassembler dans la place 2000 individus dont on précise qu'il s'agit de célibataires de 18 à 40 ans sans affectation ${ }^{34}$. Les hommes levés dans le cadre local ou les corps francs agissent souvent en lien avec les troupes régulières, comme c'est le cas en Espagne ou en Allemagne ${ }^{35}$. Il existe ainsi des actions concertées dans la Loire à la fin de mars 1814 entre partisans, gardes nationaux et des chasseurs des troupes régulières ${ }^{36}$. Mais le rapprochement avec l'armée a ses limites. Vitrolles raconte qu'à Saint-Thiébault, dans la Haute-Marne, les " paysans " ne veulent pas partager les biens pris à leurs prisonniers avec les chasseurs du général Piré venus en renfort ${ }^{37}$.

Ces troupes ont été efficaces, mais elles sont restées peu nombreuses. Il n'y a jamais eu de soulèvement général. Les prises d'armes ne concernent dans chacun des cas que quelques centaines, parfois plusieurs milliers d'hommes, soit au maximum quelques dizaines de milliers d'individus dans tout le pays. Le corps de Gustave de Damas, autour de Roanne, comprend 215 hommes environ en $1814^{38}$. Dans le Morvan, la troupe commandée par Palamède de Forbin-Janson en compte à peu près 400 en $1814^{39}$. Mais, bien évidemment, l'action suppose des complicités étendues au sein de la population, y compris de la part des notables : la cause de Wolff est défendue en 1814 auprès du feld-maréchal Schwarzenberg par le pasteur Oberlin ${ }^{40}$.

Gardes nationales et corps francs ne rassemblent pas tous les combattants. Il existe un espace en partie dépourvu de structures régulières au

30. Chambon, Pascal, La Loire..., op. cit., p. 386.

31. Les corps francs..., op. cit., p. 20.

32. Ibidem, p. 6; Nerlinger, Charles, Nicolas Wolff..., op. cit., p. 10.

33. Patel, Souvenirs des deux invasions..., op. cit., p. 30.

34. Arch. dép. de l'Aisne, A C Crépy, 4 H 2, lettre du sous-préfet de Laon aux maires de l'arrondissement, 7 juin 1815.

35. BenAERTS, Louis, Les Commissaires extraordinaires de Napoléon I er en 1814, Paris, Rieder, 1915, p. 151.

36. Chambon, Pascal., La Loire..., op. cit., p. 443.

37. Mémoires et relations politiques du baron de Vitrolles, Forgues E. (éd.), t. I, Paris, Charpentier, 1884, p. 263.

38. Chambon, Pascal, La Loire..., op. cit., p. 386.

39. Bö̈LL, Charles, Un chapitre de l'histoire d'Autun. L'année 1814, Autun, Coqueugniot, 1899, p. 16.

40. NERLINGER, Charles, Nicolas Wolff..., op. cit., p. 11-13 et 26. 
sein duquel se déclinent différents types d'actions. Il est difficile de dire de quel cadre relèvent les auteurs de telle ou telle entreprise. Dans certains lieux, c'est au sein de la garde nationale sédentaire que l'on agit, une opération concernant parfois deux ou trois localités ${ }^{41}$. Il arrive néanmoins que des groupes d'habitants, hommes et femmes, s'en prennent de leur propre initiative aux militaires étrangers. Georges Clause parle de "résistance spontanée " des paysans entre Montmirail et Sézanne suite aux exactions des cosaques en $1814^{42}$. Il se peut même que des individus isolés attaquent l'ennemi ${ }^{43}$. À cette échelle, on se situe entre action collective et organisée à l'intérieur d'un cadre, et atomisation des attitudes dans un contexte d'isolement des communautés. Ces dernières sont coupées des autorités supérieures auxquelles elles en réfèrent habituellement. Les populations réagissent en fonction d'intérêts locaux. On retrouve donc certaines des caractéristiques de l'action populaire évoquée par R. Dupuy ${ }^{44}$.

Que sait-on à présent de la dimension politique de l'engagement? Le fait de prendre les armes implique une prise de décision. Or, de nombreux facteurs vont dans le sens de l'abstention des civils. Le choc de l'invasion a paralysé nombre de Français. De plus, l'ordre de repli transmis par le pouvoir à une partie du personnel administratif constitue un mauvais exemple. Il faut aussi prendre en compte la propagande : celle contre-productive du pouvoir impérial au sujet des cosaques, et celle de l'ennemi qui prétend dissocier le sort de Napoléon de celui de ses sujets. Ceci intervient dans un contexte d'isolement des villes et des villages et d'absence d'informations fiables qui rend la prise de décision difficile. Les recrutements importants qui ont été effectués précédemment n'ont laissé que peu de recrues disponibles ${ }^{45}$. Enfin, le pouvoir napoléonien s'est employé auparavant à désarmer les civils pour en finir avec le passé révolutionnaire ${ }^{46}$.

Par ailleurs, le facteur politique pèse dans les choix. À Toulouse, le commissaire extraordinaire, le comte Louis-Marie de Caffarelli, note le 15 février 1814 que le peuple ne veut pas prendre les armes car il se souvient de la mise à l'écart des Jacobins sous le Directoire. De leur côté, les autorités redoutent le " parti de la multitude " comme le désigne Caffarelli ${ }^{47}$. Au contraire, dans la Loire, bien que les mariniers de Roanne organisés en garde nationale aient été désarmés en 1795, ils s'assemblent à nouveau avec enthousiasme en $1814^{48}$. Il est certain que le pouvoir hésite face à ces recrutements. L'empereur luimême est partagé entre la recherche de soutien et la peur d'armer le peuple.

41. FleUry, Edouard, Le département de l'Aisne..., op. cit., p. 169

42. Clause, Georges, " 1814 : la Champagne... ", art. cit., p. 264.

43. MoriceAu, Jean-Marc, " Apprendre la terre. Mémoires de Gilbert Clain (1796-1853)", Histoire et sociétés rurales, $\mathrm{n}^{\circ} 3,1995$, p. 317-318.

44. Dupuy, Roger, La Politique du peuple, XVIII ${ }^{e}-X X^{e}$ siècle, Paris, Albin Michel, 2002, p. 95.

45. BRUCHET, Max., L'Invasion et l'occupation du département du Nord par les alliés 18141818, Lille, Robbe, 1920, p. 1.

46. DeBIDour, Antonin, Étude sur le général Fabvier..., op. cit., p. 11.

47. BenAerts, Louis, Les Commissaires extraordinaires..., op. cit., p. 47.

48. Chambon, Pascal, La Loire..., op. cit., p. 441. 
Dans la Loire encore, Pascal Chambon observe que les autorités locales sont partagées à l'égard du recours aux milieux populaires ${ }^{49}$. Ces tensions sont recoupées par celles que l'on peut observer dans les localités au sujet de l'opportunité même de se battre, comme à Pontoise en $1814^{50}$.

On mesure l'implication des combattants si on la compare à l'attitude générale des populations à l'égard de l'ennemi, faite davantage de fuite, de dissimulation de biens et d'accommodements que de volonté de se battre ${ }^{51}$. À ce sujet, l'abstention peut s'expliquer de la part des cultivateurs et des éleveurs, dont les biens sont très exposés aux prélèvements et à la destruction. Par conséquent, ce sont souvent des ouvriers des bois ou des artisans moins vulnérables qui prennent les armes. Possédant moins de biens visibles, ils sont plus libres de s'engager. C'est le cas dans les Vosges, dans le Sud du Morvan et dans l'Autunois ${ }^{52}$. Le commissaire extraordinaire Chaptal, à Lyon, expose clairement ses conceptions à ce sujet le 6 janvier 1814 : les riches et les gens d'âge mûr doivent s'engager dans la garde nationale, tandis que les pauvres et les jeunes ont vocation à rejoindre les corps francs ${ }^{53}$.

L'implication individuelle est évidente. Elle s'explique d'abord par la volonté de répliquer à la violence de l'ennemi, notamment aux réquisitions et aux pillages. Le 6 mars 1814, Napoléon évoque les motivations concrètes des " paysans qui ne demandent que vengeance et à courir aux $\operatorname{armes}^{54}$ ». Un ancien des corps francs de Pontarlier explique prosaïquement que tous ont souhaité se dédommager. Sans doute pense-t-il aux sommes investies dans l'équipement et l'armement, voire à l'arrêt momentané du travail. À la fin de la campagne de 1815, on partage sous l'autorité du général Laplagne le produit de la vente des chevaux pris à l'ennemi ${ }^{55}$. Au-delà, les Morvandiaux qui se sont levés en 1814 rançonnent parfois les habitants pour vivre ${ }^{56}$. Cette attitude est compréhensible de la part d'hommes non soldés, souvent pauvres. Cependant, la majorité des partisans de Wolff refuse la solde que leur chef leur propose en $1814^{57}$. Il existe aussi une certaine éthique du combat qui exclut généralement la violence extrême.

49. Ibidem, p. 387.

50. Arch. dép. du Val-d'Oise, A M P 1 D A 19, 31.3.1814.

51. Hantraye, Jacques, Les cosaques aux Champs-Élysées, Paris, Belin, 2005, p. 99 et sq.

52. Nerlinger, Charles, Nicolas Wolff..., op. cit., p. 7; Bö̈Ll Charles, Un chapitre de l'histoire d'Autun..., op. cit., p. 16; Brosselin-Thomas, Arlette, "Les "maquis" de Bourgogne... ", art. cit., p. 97-98.

53. BENAERTS, Louis, Les Commissaires extraordinaires..., op. cit., p. 157.

54. Cité par Petiteau, Natalie, Les Français et l'Empire (1799-1815), Paris, La Boutique de l'Histoire, 2008, p. 226.

55. PAtel, Souvenirs des deux invasions..., op. cit., p. 39.

56. Brosselin-Thomas, Arlette, "Les "maquis" de Bourgogne... ", art. cit., p 98. Il arrive que des Français attaquent des étrangers afin de dérober leurs biens, mais aussi pour se venger. Un vivandier prussien du nom de Jacobi Lesser fut ainsi agressé à Étréchy le 19 juillet 1815 (Arch. dép. des Yvelines, 2 U 89, dépositions devant le tribunal d'Étampes, 25-26 et 31 juillet 1815; déposition de Lesser devant le commandant de place prussien d'Étampes, 21 juillet 1815.).

57. NERLINGER, Charles, Nicolas Wolff..., op. cit., p. 23. 
Un homme de Vailly, dans l'Aisne, est mis au ban pour avoir tué des Russes dont il était le guide. Évoquons aussi des habitants de la région de Craonne dont les proches avaient été enfumés par les Russes en mars 1814, et dont la vengeance cruelle fut dénoncée dans le pays ${ }^{58}$.

Le patriotisme constitue indéniablement un mobile de l'action chez les officiers ${ }^{59}$. La plupart des chefs de corps francs rédigent une proclamation personnelle au contenu empreint d'un patriotisme à la fois local et national, comme c'est le cas pour Gustave de Damas ${ }^{60}$. Les noms de ces unités évoquent l'attachement à la localité, tels les "Éclaireurs basques " ou les "Partisans de la Gironde " dans le Sud-Ouest ${ }^{61}$. Quelques-uns envisagent la lutte au sein des corps francs comme un avatar du combat national. A posteriori, Frantz dit défendre le sol français contre l'invasion étrangère ${ }^{62}$. Pour sa part, le colonel Fabvier combat dans l'armée régulière pendant la campagne de France, alors qu'en 1815 il rejoint la Lorraine pour organiser les corps francs ${ }^{63}$. L'adhésion de la troupe se manifeste à travers la fidélité au chef, mais on ne sait guère s'il existe une dimension idéologique. On peut parler, dans l'ensemble, d'un patriotisme des frontières. Cette dimension est attestée dans le cas du soulèvement alsacien à en croire les propos d'un témoin des combats de Wolff en 1814: "On aimait tant son pays qu'on ne pouvait supporter que l'étranger, qui en avait souillé le sol, en sortît vivant ${ }^{64}$. " Ces soulèvements concernent la plupart des zones envahies, parfois assez loin des frontières. Dans la seconde quinzaine de mars 1814, on recense des prises d'armes dans les secteurs limitrophes des départements frontaliers (Gers, Puy-de-Dôme et Saône-et-Loire, par exemple), mais aussi dans un vaste Bassin parisien allant de l'Yonne et du Loiret aux confins de l'Aisne et du Pas-de-Calais. Patriotisme et hostilité à une éventuelle Restauration se conjuguent à l'occasion. Dans les Landes, par exemple, en mars 1814, des paysans massacrent les détachements anglais et brûlent les maisons des royalistes ${ }^{65}$. Des textes contemporains de la campagne de France évoquent la crainte du rétablissement des dîmes et de l'Ancien Régime $^{66}$. Ceci s'accompagne du retour des chants révolutionnaires, que chantent ouvertement des militaires. C'est ainsi que les troupes, régulières françaises entrent à Arcis-sur-Aube à la fin de février 1814 au son du " ça ira ", que l'on n'avait pas entendu depuis longtemps ${ }^{67}$.

58. FleURY, Edouard, Le Département de l'Aisne..., op. cit., p. 317-318 et 322 et Les corps francs..., op. cit., p. 30.

59. NERLINGER, Charles, Nicolas Wolff..., op. cit., p. 37-40.

60. Chambon, Pascal, La Loire..., op. cit., p. 420.

61. Lauzun, Philippe, Florian et ses bandes de partisans..., op. cit., p. 4 et 6.

62. Pétition..., op. cit., p. 1.

63. DeBIDOUR, Antonin, Étude sur le général Fabvier..., op. cit., p. 14.

64. On ignore toutefois s'il ne s'agit pas en partie d'une reconstruction postérieure à

1871 (NERLINGER, Charles, Nicolas Wolff..., op. cit., p. 20).

65. HoussaYe, Henry, 1814 ..., op. cit., p. 404.

66. MÉNAGER, Bernard, Les Napoléon du peuple, Paris, Aubier, 1988, p. 26.

67. JAQUOT, "Souvenirs de l'invasion de 1814 à Arcis-sur-Aube ", L'Arcisien, Arcis, Frémont, 1876, p. 74 . 
Les prises d'armes ne se résument pas au combat contre l'ennemi. Il existe en outre des manifestations d'opposition au pouvoir impérial, en particulier au sujet de la conscription. Région frontalière n'est donc pas toujours synonyme de patriotisme. Au début de 1814, dans le Nord, le Pas-de-Calais et le Sud de l'actuelle Belgique, un mouvement s'appuyant sur le rejet de la conscription et des impôts indirects entraîne la constitution de bandes armées $^{68}$. Le chef est Louis Fruchart (1791-1851), dit Louis XVII, issu d'une famille de cultivateurs royalistes de la région d'Hazebrouck ${ }^{69}$. Il parvient à tenir une grande partie du Nord et du Pas-de-Calais à la fin de janvier $1814^{70}$, avant de se rapprocher des troupes alliées du colonel de Geismar, au service du duc de Saxe-Weimar ${ }^{71}$. Outre cet exemple, des levées contre la conscription sont signalées dans l'Ouest, comme dans les Deux-Sèvres, la LoireInférieure et la Vendée ${ }^{72}$, dans les secteurs concernés par les troubles des années 1790. Les milieux aisés sont aussi impliqués dans ce type d'action. En 1815, le comte Hippolyte d'Espinchal, un ancien émigré, constitue à Lyon un corps de 1100 hommes nommé "Les chasseurs d'Henri IV ", destiné à tenir la ville au nom du roi. Il recrute des commerçants, des propriétaires et des jeunes gens ayant appartenu à la Maison du Roi ${ }^{73}$.

Ces différentes manifestations permettent de distinguer une France de l'Ouest et du Nord, où les levées sont le fait de royalistes ou d'opposants à la conscription, et celle de l'Est, plus favorable à l'empereur. Néanmoins, il ne faut pas négliger des secteurs où l'on peut observer des attitudes contrastées : en mars-avril 1814, on constitue dans la Dordogne un corps d'employés des douanes et d'autres administrations réfugiés de la Gironde, tandis que près de Sarlat a lieu un rassemblement de paysans opposés à la conscription. Dans un tel contexte, on comprend que la décision d'armer le peuple puisse être interprétée comme un test de fidélité. Dans le département des Forêts, par exemple, le 8 janvier 1814, le commissaire extraordinaire dans la $3^{\mathrm{e}}$ division militaire, Charles-Antoine Chasset, renonce à organiser les levées car il craint que la population ne se retourne contre les Français ${ }^{74}$. Quel que soit le camp adopté, la volonté de combattre suppose un débat. À Pontarlier en 1814, même si le soulèvement n'a pas lieu, on discute longuement à ce sujet. Le combat est déclenché lors de l'invasion suivante, dans le cadre des corps francs. Un commandant est alors élu par acclamation, expérience démocratique qui rappelle l'élection des officiers au cours des premiers temps de la Révolution ${ }^{75}$. Lors de la Restauration, ces corps francs inquiètent le pouvoir royal, d'où la décision de les supprimer qui survient dès le 6 mai en 1814, et

68. Bruchet, Max, L'Invasion et l'occupation du département du Nord..., op. cit., p. 2-3.

69. FAUCHILLE, Paul, Une Chouannerie flamande au temps de l'Empire (1813-1814), Paris, Pedone, 1905, p. 45-46 et 49.

70. Ibidem, p. 85.

71. Ibid., p. 238 et 250 .

72. BENAERTS, Louis, Les Commissaires extraordinaires..., op. cit., p. 66-68.

73. Chambon, Pascal, La Loire..., op. cit., p. 495-496.

74. Benaerts, Louis, Les Commissaires extraordinaires..., op. cit., p. 188, 191 et 10.

75. PAtEl, Souvenirs des deux invasions..., op. cit., p. 24, 30 et 36-37. 
le 20 juillet en 1815. Le pouvoir se montre plus méfiant à l'égard des combattants en 1815 en leur ordonnant d'abandonner leurs armes et de rentrer chez eux sans rejoindre les troupes de ligne comme en $1814^{76}$.

\section{La postérité des prises d'armes}

Dès les Cents-Jours, le pouvoir tente de récupérer ces actions à son profit. Napoléon honore quelques combattants des troupes auxiliaires par la remise de la Légion d'honneur et les présente comme ses partisans. Le 16 mars 1815, par exemple, il distingue Hubinet, maître de poste à Chissey, dans le Morvan ${ }^{77}$. En cela, il reprend un mouvement engagé avant la première abdication. Ainsi, après la dissolution de son corps franc, Wolff passe au service direct de Napoléon à la fin de la campagne de France, avant de se voir promu colonel et décoré de la Légion d'honneur ${ }^{78}$.

La dimension politique des prises d'armes doit être envisagée dans la durée. Les premières années de la seconde Restauration sont une période d'intense expression politique populaire, notamment sous la forme de rumeurs. On recense beaucoup de militaires parmi les individus colportant la fausse nouvelle du retour de Napoléon, par exemple ${ }^{79}$. Les années qui suivent le retour des Bourbons s'accompagnent de tentatives de contestation du pouvoir dans lesquelles les militaires jouent un rôle essentiel. Elles s'inscrivent dans un contexte de recomposition de l'armée qui s'accompagne de la mise à l'écart et de la fragilisation de beaucoup d'officiers, les demi-soldes. En arrière-fond a lieu la Terreur blanche. J'insisterai plus particulièrement sur le lien entre les prises d'armes de 1814 et 1815 et les complots militaires des années 1816-1822.

Les principales actions ont lieu sous la Seconde Restauration, alors que les troupes alliées sont présentes dans les départements du Nord Est et de l'Est afin de garantir la stabilité du régime de Louis XVIII. La France bonapartiste est celle des départements de l'Est, terrain privilégié des levées de 1815, même si l'Ouest est concerné, avec un complot dans le Maine-etLoire $^{80}$. Les premières actions sont surtout le fait d'anciens officiers de la Révolution et de l'Empire, comme le complot du Bazar français, fomenté par des militaires et des étudiants bonapartistes et républicains ${ }^{81}$. Les tentatives suivantes impliquent au contraire des catégories plus larges de la société. Leur organisation rappelle celle des prises d'armes de 1814 et 1815. On évoquera ici les principaux traits de quelques-unes de ces affaires.

76. Bulletin des Lois, $\mathrm{n}^{\circ} 12$ (6 mai 1814) et 7 (20 juillet 1815). Les flux d'anciens soldats sont mal perçus ; voir, par exemple, Arch. dép. de l'Aisne, A C Crépy, 4 H 2, lettre du souspréfet de Laon aux maires, 3 juillet 1815.

77. BoËLL, Charles, Un chapitre de l'histoire d'Autun..., op. cit., p. 29.

78. NERLINGER, Charles, Nicolas Wolff..., op. cit., p. 28.

79. MÉNAGer, Bernard, Les Napoléon..., op. cit., p. 20. Ploux, François, De Bouche à oreille. Naissance et propagation des rumeurs dans la France du XIXe siècle, Paris, Aubier, 2003.

80. Ibidem, p. $45,47-48$ et 51 .

81. Ibid., p. 26 et n. 70 p. 370. 
La conspiration de Didier, à Grenoble, du nom de l'un de ses principaux responsables, avocat et directeur de l'école de droit de la ville, rassemble beaucoup d'anciens soldats et de sous-officiers retraités ou en demi-solde, mais elle concerne également des civils ${ }^{82}$. Ces hommes tentent un coup de main sur le chef-lieu de l'Isère le 4 mai $1816^{83}$. Outre l'appel aux habitants des campagnes, on retrouve des caractéristiques observées précédemment. Signalons par exemple la présence de douaniers et de gardes forestiers, dont un ancien des corps francs ${ }^{84}$. Enfin, lors de son procès, Didier évoque le village de Saint-Chaffrey, dans les Hautes-Alpes, dont les habitants ont défendu un fort de Briançon en $1815^{85}$. Le complot de Lyon en 1817 a un caractère plus complexe, car il s'agit en partie d'une provocation des autorités militaires et policières. Il n'empêche que le projet rassemble beaucoup d'intervenants de bonne foi. L'affaire implique des officiers à la demi-solde, dont un nommé Gaslon qui fut chef de corps francs pendant les Cent Jours ${ }^{86}$. Ces militaires s'appuient sur des paysans qui se soulèvent au cri de «Vive l'Empereur $^{87}$ ". Un autre complot a lieu dans l'Est de la France à la fin de 1821 et au début de $1822^{88}$. Déclenché à Belfort au sein du $29^{\mathrm{e}}$ régiment de ligne, les organisateurs en sont des militaires liés à des personnalités politiques, dont Lafayette, le député libéral Manuel et Nicolas Koechlin. Ce dernier, grand industriel alsacien, a servi en 1814 en tant que colonel de la garde nationale. On retrouve Joseph Brice, avec des hommes de son corps franc de $1815^{89}$. Autre parcours, celui du colonel Fabvier, également ancien des corps francs. Déçu par la Restauration, il dénonce la présence des Alliés en France, ce qui le conduit à prendre part aux affaires du Bazar français et de Belfort. Sa démarche acquiert une dimension européenne lorsqu'il s'oppose à l'expédition d'Espagne, avant de se battre en Grèce entre 1823 et 1828. Il revient en France et lutte encore à Paris pendant les journées de juillet $1830^{90}$.

À côté de ces complots impliquant principalement des officiers supérieurs et des personnalités politiques, il existe des formes d'action essen-

82. Gullon, Édouard, Les Complots militaires sous la Restauration, Paris, Plon, 1895, p. 84-85.

83. REY, Joseph, Histoire de la conspiration de Grenoble, Grenoble, J.-L. Barnel, 1847, p. 113. 84. Ibidem, p. 78-79.

85. Ducoln, Auguste, Paul Didier. Histoire de la conspiration de 1816, Paris, Dentu, 1844, p. 151 ; REY, J., Histoire de la conspiration de Grenoble..., op. cit., p. 179.

86. GuILlon, Édouard, Les Complots militaires..., op. cit., p. 96-97.

87. MÉnager, Bernard, Les Napoléon..., op. cit., p. 22.

88. BlaISON, Louis, Une Ville de garnison sous la Restauration : le complot militaire de Belfort, 1822, Paris, Berger-Levrault, 1914, p. 4 et 34.

89. Gulluon, Édouard, Les Complots militaires..., op. cit., p. 157, 159, 161 et 165; BLAISON Louis, Une Ville de garnison..., op. cit., p. 4. Enfin, signalons pour mémoire le complot de Saumur, en 1822, qui devait impliquer des anciens soldats et des paysans-mariniers de la Loire, autour du général Berton avec la participation de la troupe et de gendarmes (Gulllon, Édouard, Les Complots militaires..., op. cit., p. 183, 187 et 202; GAUCHAIS, colonel, Histoire de la conspiration de Saumur, Paris, l'auteur, 1832, p. 38 et 40).

90. Debidour, Antonin, Étude sur le général Fabvier..., op. cit., p. 15 et 21-23 et 26-32 et Les Corps francs..., op. cit., p. 3 et 33. Sur le rôle des anciens officiers des guerres de la Révolution et de l'Empire dans les mouvements politiques en Europe, voir BRUYÈREOstells, Walter, La Grande armée de la liberté, Paris, Tallandier, 2009. 
tiellement populaires. Citons une prise d'armes qui eut lieu en 1816 dans le Sud de la Sarthe. Six mois après la chute de Napoléon, un petit groupe de journaliers, de meuniers et de petits propriétaires de la région du Lude tente de s'emparer des armes de cultivateurs des environs, souhaitant confusément aller plus loin. De façon significative, ils se donnent un nom évoquant les corps francs, "Les Vautours de Bonaparte ${ }^{91}$ ". Dans cette affaire se mêlent des enjeux strictement locaux (les conflits au sein des élites), départementaux - l'un des comploteurs conçut en 1815 le projet de constituer un corps franc contre les Prussiens - et enfin nationaux avec l'enrôlement de plusieurs protagonistes dans l'armée en 1815. Ce mouvement est en lien avec la politisation des masses, puisqu'il intervient à la suite de l'élection des maires et adjoints, moment d'intense débat pendant les Cents-Jours. Toutefois, il est difficile de dire dans quelle mesure l'expérience des corps francs a débouché sur une volonté de contestation étendue. Les individus mêlés aux conspirations de la Restauration sont en effet peu nombreux parmi les 20000 officiers en demi-solde que compte la France vers $1815^{92}$.

En tout cas, signe du caractère politique attribué à leur action, nombre de combattants des corps francs ont fait l'objet de mesures de police et de sanctions judiciaires sous la Restauration. Frantz connaît ainsi seize années d'exil en Prusse à la suite d'une condamnation à mort ${ }^{93}$. En juillet 1816, le colonel de cavalerie Viriot est incarcéré à Nancy. Ayant levé un corps franc en Lorraine pendant les Cents-Jours, il est accusé d'avoir comploté par la suite contre les troupes alliées ${ }^{94}$. Appartenant à des milieux plus modestes, les Vautours de Bonaparte sont brutalement sanctionnés par la Cour prévôtale de la Sarthe en 1816 : quatre d'entre eux sont guillotinés et dix autres sont incarcérés ${ }^{95}$. C'est la Monarchie de juillet qui met fin à ces persécutions. Brice se voit ainsi acquitté par le conseil de guerre de Metz en 1830, avant d'être réintégré la même année dans l'armée et de finir général de brigade en $1848^{96}$. Fabvier est également honoré sous la Monarchie de juillet, qui le nomme pair de France ${ }^{97}$.

Quant à la postérité littéraire de cet engagement, elle apparaît tardivement. Sous le Second Empire, une littérature de second ordre célèbre les exploits de paysans qui se sont défendus eux-mêmes. C'est, en 1869, $L a$ Chasse aux Cosaques ou la Champagne en 1814, de M. de Robville, ou Les Alliés en Champagne - 1814 -, de Narcisse Blanpain ${ }^{98}$. Au même moment,

91. HANTRAYE, Jacques, Les Vautours de Bonaparte, mémoire de maîtrise, dir. A. Corbin, Université de Paris I, 1992.

92. VIDALENC, Jean, Les demi-solde. Étude d'une catégorie sociale, Paris, Rivière, 1955, p. $197-198$ et 33 .

93. Pétition..., op. cit., p. 6-7.

94. Précis pour P. F. Viriot, Metz, Lamort, 1816, p. 5 et 8.

95. HANTRAYE, Jacques, Les Vautours..., op. cit., p. 113-115.

96. Les corps francs du commandant Brice, op. cit., p., 25-26.

97. Debidour, Antonin, Étude sur le général Fabvier..., op. cit., p. 36 et Vidalenc, Jean, Les demi-solde..., op. cit., p. 202.

98. Paris, Lebailly, 1869, p. 7 pour le premier, Paris, Vanier, 1869, p. 258 pour le second. 
l'aventure des corps francs est magnifiée par les écrivains Emile Erckmann et Alexandre Chatrian, tous deux nés dans l'Est de la France quelques années après la chute de l'Empire, qui ont magistralement évoqué ces faits dans le roman intitulé L'Invasion, paru en 1862.

Les prises d'armes survenues lors des invasions de 1814 et 1815 sont inégalement réparties dans l'espace, même si elles sont plutôt concentrées dans l'Est pour les partisans de l'empereur et dans l'Ouest pour les opposants à la conscription. Elles soulèvent la question des motivations des participants, parfois difficiles à appréhender. Elles n'ont pas toujours revêtu un caractère politique. Il peut s'agir tout simplement de se défendre ou de se venger, sans négliger le lien avec le brigandage.

Les levées ont beau être parfois spontanées, elles sont le plus souvent encadrées par le pouvoir et les élites. On relève une forte présence de militaires, car de nombreux Français ont acquis auparavant une expérience de l'armée. Les combattants sont enrôlés dans des structures très militarisées. C'est bien l'intensification du rapport entre l'individu et l'État qui est ici en jeu. Le rôle des officiers est important, notamment sur le plan idéologique.

L'engagement témoigne souvent d'une adhésion individuelle. Les corps francs valorisent l'initiative, soldats et officiers se persuadant qu'ils peuvent sauver le pays par leur action. Ici réside une dimension politique essentielle. Sans doute ceci est-il en rapport avec le bonapartisme conçu comme l'action volontariste d'un homme.

Les levées indiquent l'existence de réseaux sociaux et politiques, ce qui explique qu'elles sont redoutées par le pouvoir - même lorsque celui-ci en bénéficie-, d'autant plus que ce type d'action représente une transgression par rapport au combat classique. On observe des solidarités horizontales dans la lutte et lors de la répression, mais aussi à travers la fidélité à des chefs qui paient doublement de leur personne, lors du combat et par la suite. Les levées constituent indéniablement un test de fidélité à l'égard du pouvoir. Toutefois, le fait que les hommes manifestent apparemment plus d'attachement envers leurs chefs que d'adhésion à des idées constitue une limite de ces prises d'armes.

Après le retour des Bourbon, ces actions trouvent leur prolongement pendant la Restauration, en particulier sous la forme de complots politiques et militaires. Il convient donc d'envisager la Restauration sous l'angle de la continuité des options politiques et des fidélités personnelles au sein des forces de contestation. Dans cette optique, il faut intégrer l'influence de l'expérience combattante de 1814-1815 sur les prises d'armes de 18161822. Ces dernières n'apparaissent pas seulement comme des échecs, mais aussi comme des moments de politisation dans un temps où le jeu électoral est circonscrit à quelques catégories sociales, et l'opinion étroitement surveillée. 
En ce sens, les années 1814 et 1815 apparaissent comme un moment complexe car la prise d'armes n'est pas seulement dirigée contre l'ennemi, mais elle débouche également sur une contestation du pouvoir. D'un combat à un autre, il n'y a qu'un pas, qu'une minorité a franchi.

\section{RÉSUMÉ}

Les prises d'armes liées aux invasions de 1813-1815 en France sont mal connues, car peu documentées. De plus, les hommes qui les ont animées ont souffert rétrospectivement d'un discrédit sous la Restauration. Organisées dans le cadre de la garde nationale ou des corps francs, ou bien spontanées, elles caractérisent surtout les régions menacées. Très marquées par la personnalité de chefs dotés d'un grand charisme, elles réunissent au mieux quelques centaines d'hommes. Généralement favorables à l'Empire, les combattants agissent parfois en opposition à la conscription. On ne peut négliger le fait que le souvenir de ces soulèvements a influé sur les prises d'armes et les complots militaires intervenus sous la Restauration. La littérature romanesque de la seconde moitié du $\mathrm{XIX}^{\mathrm{e}}$ siècle a contribué à revaloriser ces épisodes militaires oubliés.

\section{ABSTRACT}

The uprisings which occurred in France at the end of the Napoleonic era are now little known, owing to the lack of books and original documents upon the subject. Closely linked with the invasion by the allied armies between 1813 and 1814 , most of them took place close to the eastern boundaries. Spontaneous or organized within the militia, they were generally headed by charismatic individuals, generally young officers. Though they were much despised by the regular army and prosecuted by the civil authorities under the monarchy, they were eventually highly celebrated during the late 19th century by novelists and local historians. 